\title{
Etude expérimentale de la propagation des trains d'ondes en zone côtière
}

\author{
Iskander ABROUG ${ }^{1,2}$, Nizar ABCHA ${ }^{2}$, Armelle JARNO ${ }^{1}$, François MARIN ${ }^{1}$ \\ 1. Normandie Université, UNILEHAVRE, CNRS, UMR 6294 LOMC, 76600 Le Havre, \\ France. \\ iskander.abroug@unicaen.fr ; armelle.jarno-druaux@univ-lehavre.fr ; \\ françois.marin@univ-lehavre.fr \\ 2. Normandie Université, UNICAEN, UNIROUEN, CNRS, UMR 6143 M2C, 14000 \\ Caen, France. \\ nizar.abcha@unicaen.fr
}

\section{Résumé :}

Notre travail repose sur une étude expérimentale de la propagation des trains d'ondes fortement non-linéaires se propageant en eau intermédiaire vers la zone côtière. Trois méthodes de génération ont été employées. Ces méthodes dépendent du spectre à partir duquel les trains d'ondes sont construits. Il s'agit du spectre gaussien, spectre de PiersonMoskowitz et spectre de JONSWAP. Les essais ont été réalisés dans le canal à houle du laboratoire M2C (UMR 6143 Caen). Les signaux obtenus par les sondes résistives sont utilisés pour la détermination de l'évolution spatiale des transferts énergétiques et des interactions non-linéaires entre les composantes fréquentielles.

Un partitionnement spectral a été effectué sur chaque spectre de référence pour quantifier les variations spatiales de l'énergie dans chaque gamme de fréquences. Des transferts énergétiques en amont et en aval de déferlement ont été mis en évidence et ensuite quantifiés. Une approche bispectrale par ondelettes a été utilisée dans le but de quantifier les interactions non-linéaires entre les composantes fréquentielles grâce à un paramètre appelé bicohérence. Au cours de la propagation, ce paramètre augmente progressivement et atteint des valeurs proches de 1 au moment du déferlement et décroit drastiquement en aval de ce déferlement.

\section{Mots-clés :}

Trains d'ondes, Energie spectrale, Bicohérence, JONSWAP, Pierson-Moskowitz. 


\section{Thème 1 - Hydrodynamique côtière}

\section{Introduction}

La surface réelle de la mer peut être décrite comme la superposition de nombreux paquets d'ondes focalisant qui se propagent dans différentes directions avec différentes vitesses. La focalisation dispersive est la focalisation spatio-temporelle d'un train d'ondes modulé en fréquence. Ce phénomène est également connu pour avoir joué un rôle important dans la formation des vagues extrêmes (TOUBOUL \& KHARIF, 2015). Les ondes longues se propagent à une vitesse plus importante que les ondes courtes situées initialement devant. Par conséquent des vagues d'amplitudes importantes se forment en un point qu'on appelle le point de focalisation (TOUBOUL \& KHARIF, 2006). En se basant sur la théorie linéaire, l'élévation de surface libre pour un train d'ondes peut s'écrire comme suit :

$\eta(x, t)=\sum_{i=1}^{n} a_{i} \cos \left(k_{i} x-w_{i} t+\Phi_{i}\right)$

où $a_{i}$ est l'amplitude de la ième composante fréquentielle, $k_{i}$ est le nombre d'onde, $\omega_{\mathrm{i}}=2 \pi f_{i}$ est la pulsation et $\Phi_{\mathrm{i}}$ est la phase à la focalisation. Dans la littérature nous distinguons plusieurs méthodes de génération de trains d'ondes. Ces méthodes dépendent principalement du spectre à partir duquel ces trains d'ondes sont construits. Dans ce travail nous allons nous focaliser particulièrement à l'étude de 3 paquets d'ondes construits à partir du spectre: Gaussien, Pierson-Moskowitz et JONSWAP. Dans la littérature, la plupart des recherches se focalisent sur l'évolution des trains d'ondes se propageant dans une eau de profondeur constante (TIAN et al., 2011). Cependant, il est à souligner que l'influence de la variation de la profondeur d'eau a été moins étudiée dans la caractérisation spatio-temporelle des trains d'ondes (MADSEN \& SORENSEN, 1993). Dans ce qui suit nous proposons une étude expérimentale de la transformation des trains d'ondes se propageant dans une eau de profondeur intermédiaire jusqu'au rivage. Cette transformation est caractérisée par deux approches différentes :

(1) Approche spectrale : il s'agit d'une analyse fine des échanges énergétiques lors de la propagation du train d'ondes.

(2) Approche bispectrale : il s'agit d'identifier les interactions non-linéaires entre les composantes fréquentielles grâce aux mesures de bicohérence (triades résonantes).

\section{Matériel et méthodes}

\subsection{Matériel}

Le canal à houle du laboratoire Morphodynamique Continentale et Côtière de Caen dans lequel tous les essais ont été menés possède une longueur $\mathrm{L}=20 \mathrm{~m}$, une largeur $1=0.8 \mathrm{~m}$. Un générateur de vagues de type piston est utilisé pour simuler de nombreuses conditions de houles. Les vagues se propagent vers l'autre extrémité du canal, de gauche à droite, selon la figure 1, à travers une profondeur d'eau uniforme jusqu'à une plage en PVC 


\section{XVİ̀mes Journées Nationales Génie Côtier - Génie Civil \\ Le Havre 2020}

inclinée $(\beta=1 / 25)$ qui se situe à 9.5 mètres du générateur. La hauteur d'eau retenue est $h_{0}=0.3 \mathrm{~m}$. Sur la figure $1, x=0$ correspond à la position moyenne du batteur.

Les trains d'ondes sont générés en imposant une fréquence de pic $f_{p}$ et une distance de focalisation $x=12 \mathrm{~m}$ basée sur une focalisation linéaire dans une eau de profondeur constante. Il est important de souligner que le déferlement sur la pente empêche la focalisation parfaite, ce qui n'est pas l'objectif ultime de ce travail (WHITTAKER et al., 2017). La présence de la pente et le phénomène de focalisation prématurée (LIU et al., 2016) engendrent la présence d'un deuxième déferlement qui se produit en aval du premier. Nous nous appellerons la distance qui sépare les deux déferlements, zone de déferlement $x b$. Le paramètre $f_{p}$ est choisi de telle sorte que $k_{p} h_{0} \sim 0.9$ et par la suite l'hypothèse de propagation du train d'ondes dans une eau de profondeur intermédiaire vers la zone côtière est bien vérifiée $\left(k_{p}:\right.$ le nombre d'onde correspondant à $f_{p}$ ). Pour tous les trains d'ondes générées, les élévations de surface libre ont été enregistrées à différents endroits le long du canal avec deux sondes résistives (voir figure 1). En effet, la sonde 1 est fixée en $x=4 \mathrm{~m}$ et utilisée comme une référence temporelle. La sonde 2 est déplacée de $20 \mathrm{~cm}$ après chaque test jusqu'à la dernière acquisition située en $x=14 \mathrm{~m}$ et par la suite, chaque train d'ondes est étudié sur une distance de $10 \mathrm{~m}(50 \times 0.2=10 \mathrm{~m})$. La durée de chaque signal temporel est $\Delta T=35 \mathrm{~s}$ avec un pas de temps $\Delta t=0.02 \mathrm{~s}$. Les 4 trains d'ondes générés possédent une non-linéarité similaire $S_{0} \sim 0.23$ (voir tableau 1).

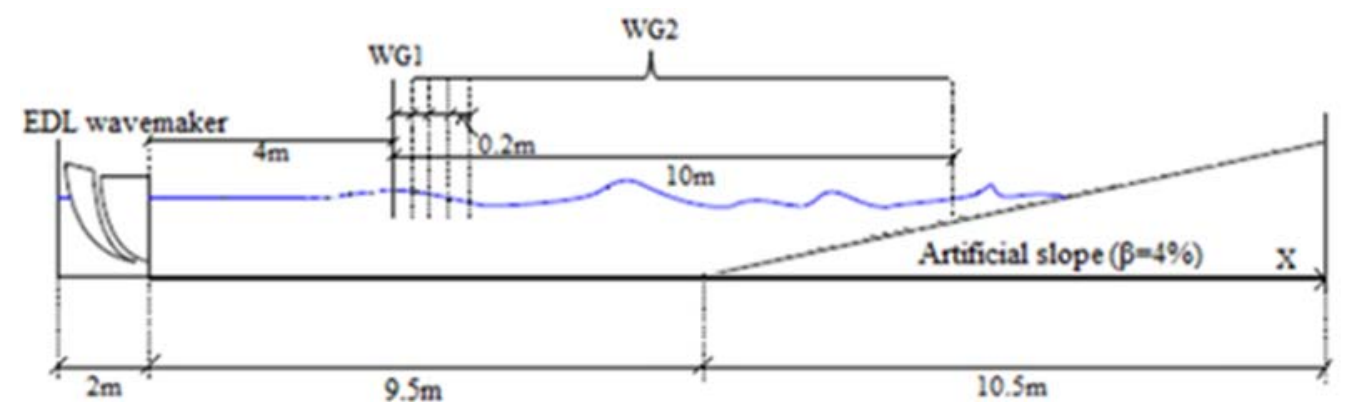

Figure 1. Configuration expérimentale. WG1 et WG2 désignent les deux sondes utilisées.

Tableau 1. Caractéristiques des trains d'ondes étudiés.

\begin{tabular}{llll}
\hline Trains d'ondes & Spectre & $\boldsymbol{f}_{\boldsymbol{p}}(\mathbf{H z})$ & $\boldsymbol{x}_{\boldsymbol{b}}(\boldsymbol{m})$ \\
\hline GW1 & Gaussien & 0.69 & {$[11.70 ; 12.70]$} \\
GW2 & Pierson-Moskowitz & 0.66 & {$[12.65 ; 13.28]$} \\
GW3 & Jonswap $(\gamma=3.3)$ & 0.75 & {$[12.13 ; 12.81]$} \\
GW4 & Jonswap $(\gamma=7)$ & 0.75 & {$[12.07 ; 12.69]$} \\
\hline
\end{tabular}




\section{Thème 1 - Hydrodynamique côtière}

\subsection{Méthodes}

\subsubsection{Approche spectrale}

Pour quantifier les changements spectraux lors de la propagation du train d'ondes sur le fond plat et sur la plage, en amont et aval de déferlement, une étude spectrale détaillée s'est imposée. Dans cette optique, chaque spectre de référence calculé en $x=4 \mathrm{~m}$ est partitionné en 4 différentes régions fréquentielles, voir figure 2 (ABROUG et al., 2020) :

- E1 : région du pic central ;

- E2 : région de transfert;

- E3 : région des hautes fréquences ;

- $\mathrm{E} 4$ : région des basses fréquences.

E0 représente l'énergie totale contenue dans l'intervalle $f \in\left[0.2 \mathrm{~Hz}, 3 f_{p}\right]$ et l'énergie est considérée comme négligeable en dehors de E0, E1 à E4 (<1\% de E0).

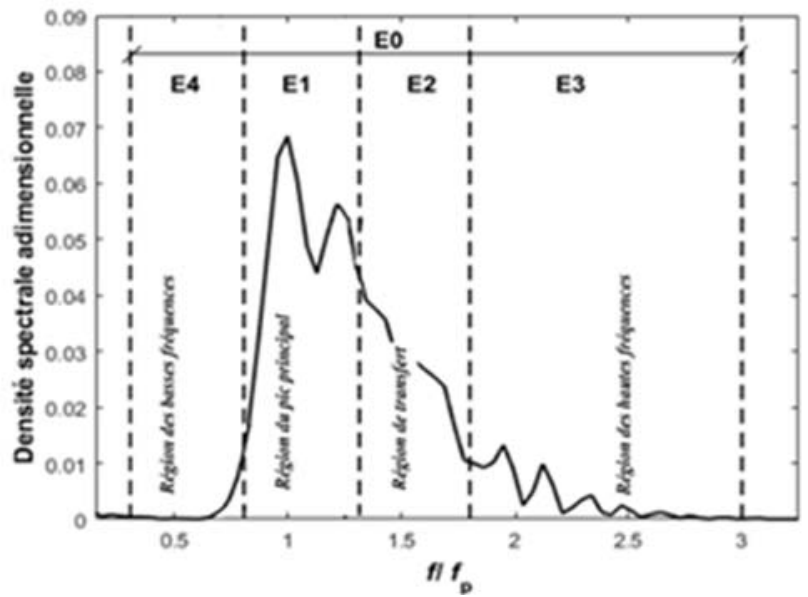

Figure 2. Partitionnement d'un spectre d'un train d'ondes de Pierson-Moskowitz.

\subsubsection{Approche bispectrale}

La notion d'énergie spectrale décrite dans la section précédente permet d'avoir la distribution de l'énergie dans le domaine fréquentiel mais, ne donne pas d'information sur le couplage de phase entre les composantes. Ainsi, le paramètre de bicohérence est utilisé dans ce travail pour détecter l'évolution spatiale du couplage quadratique de phase entre les composantes au cours du processus de focalisation.

Dans la littérature, nous distinguons deux approches pour le calcul de la bicohérence. La première est basée sur l'analyse de Fourier (ELGAR \& GUZA, 1988 ; ELDEBERKY, 1996 ; BECQ-GIRARD et al., 1999). Plus récemment, DONG et al. (2008) ont démontré, en se basant sur une analyse de Fourier, qu'un intervalle de confiance important $(95 \%)$ exige au moins 100 degrés de liberté (i.e. long signal) pour obtenir une bicohérence raisonnable (CHUNG \& POWERS, 1998). Pour cette raison, cette approche ne peut pas être utilisée pour étudier le couplage de phase entre les composantes fréquentielles dans 


\section{XVIèmes Journées Nationales Génie Côtier - Génie Civil \\ Le Havre 2020}

un signal relativement court (LARSEN et al., 2001). La deuxième approche correspond à une démarche basée sur les ondelettes. Cette méthode, qui représente un outil puissant pour les signaux temporels relativement courts, est choisie par la suite pour étudier les interactions non-linéaires entre les composantes fréquentielles dans le cas de nos essais. La transformée en ondelettes continue d'un signal temporel $f(t)$ est définie comme suit :

$W T(a, \tau)=\int_{-\infty}^{+\infty} f(t) \psi_{a, \tau}^{*} d t$

où $\psi a, \tau$ représente l'ondelette mère, $a$ est le facteur de dilatation, $\tau$ est le facteur de translation et * correspond au complexe conjugué. Nous avons choisi l'ondelette de Morlet en raison de sa nature oscillatoire et de la proche relation entre l'échelle pseudo temporelle $a$ et la période de Fourier (i.e. T a) (MA et al., 2010). Mathématiquement, l'ondelette de Morlet s'écrit comme un produit d'une onde sinusoïdale et une enveloppe gaussienne (FARGE, 1992). Du point de vue pratique, pour calculer $W T(a, \tau)$ d'une série temporelle échantillonnée, les deux paramètres $a$ et $\tau$ doivent être discrétisés (TORRENCE \& CAMPO, 1998) :

$a_{i}=a_{0} 2^{i \delta}, i=0,1,2, \ldots, M$

$M=\frac{1}{\delta} \log _{2}\left(\frac{N \Delta t}{a_{0}}\right)$

où $\Delta t$ le temps d'échantillonnage, $a_{0}=2 \Delta t$ le facteur de dilatation le plus petit, $\mathrm{M}$ la plus grande échelle et $\delta$ le facteur d'échelle (TORRENCE \& COMPO, 1998). Ce dernier paramètre doit être bien choisi pour fournir un échantillonnage adapté au facteur de dilatation $a$. Il est aussi important de souligner qu'un facteur d'échelle petit fournit une résolution élevée mais une vitesse de calcul lente. Nous avons opté pour un facteur d'échelle $\delta=0.02<<0.5$ qui nous permettra d'avoir des hautes résolutions de calcul. Nous avons travaillé un vecteur qui contient 395 facteurs de dilatation allant de 0.04 pour la détection des plus hautes fréquences à 11.83 pour la détection des plus basses fréquences. Les interactions entre les composantes fréquentielles sont supposées se faire par triades résonantes. Une mesure du terme de corrélation du $3^{\text {ème }}$ ordre normalisé par la densité bispectrale (Eq. 5), appelée bicohérence (Eq. 6), est alors possible à partir de l'élévation de surface libre à une abscisse donnée.

$B\left(a_{1}, a_{2}\right)=\int W T_{x}\left(a_{1}, \tau\right) W T_{x}\left(a_{2}, \tau\right) W T_{x}^{*}\left(a_{3}, \tau\right) d \tau$

$b^{2}\left(a_{1}, a_{2}\right)=\frac{\left|B\left(a_{1}, a_{2}\right)\right|^{2}}{\left[\int_{t=0}^{t=35}\left|W T_{x}\left(a_{1}, \tau\right) W T_{x}\left(a_{2}, \tau\right)\right|^{2} d \tau\right] \int_{t=0}^{t=35}\left|W T_{x}\left(a_{3}, \tau\right)\right|^{2} d \tau}$

Ce paramètre varie entre 0 pour une interaction non-linéaire inexistante et 1 pour une interaction non-linéaire maximale. Le paramètre de bicohérence permet de mesurer, dans 


\section{Thème 1 - Hydrodynamique côtière}

un intervalle de temps fini, l'interaction entre trois composantes d'ondelettes d'échelle pseudo fréquentielle $a_{1}, a_{2}$ et $a_{3}$ de telle sorte que les deux conditions suivantes sont remplies (VAN MILLIGEN et al., 1995) :

$\frac{1}{a_{3}}=\frac{1}{a_{1}}+\frac{1}{a_{2}}$

$\varphi_{3}=\varphi_{1}+\varphi_{2}+$ constante

où $a_{1}, a_{2}$ et $a_{3}$ désignent les échelles pseudo fréquentielles et $\varphi_{1}, \varphi_{2}$ et $\varphi_{3}$ sont les phases correspondantes. Le couplage quadratique non-linéaire se produit entre $a_{1}$ et $a_{2}$, ainsi il génère une troisième composante $a_{3}$. La relation entre l'échelle et la fréquence (i.e. $a=1 / f$ ) permet d'interpréter la densité bispectrale comme un couplage de phases entre les fréquences $f_{1}, f_{2}$ et $f_{3}=f_{1}+f_{2}$ (LARSEN et al., 2001).

\section{Résultats}

\subsection{Transferts énergétiques}

Les bornes des bandes fréquentielles varient, parfois d'une manière remarquable, en fonction du spectre considéré. L'idée était de réaliser un partitionnement spectral sur des trains d'ondes se propageant dans une eau de profondeur intermédiaire $k h \sim 0,9$ tout en utilisant la même technique (ABROUG et al., 2020). Le partitionnement en région spectrales est présenté sur la figure 3 dans le cas d'un train d'ondes de JONSWAP (GW3). Une étude spectrale détaillée a été menée sur les signaux temporels obtenus entre $x=4 \mathrm{~m}$ et $x=14 \mathrm{~m}$. Nous avons réussi à mettre en évidence des transferts énergétiques entre les différentes régions au cours de la propagation du train d'ondes.

La quantité initiale de l'énergie située dans E1 varie entre 50\% et 70\% de l'énergie totale. Ces pourcentages sont qualitativement comparables à ceux obtenus dans les travaux de TIAN et al., (2011). Concernant les deux trains d'ondes de JONSWAP, l'énergie dans E1 reste relativement constante le long du fond plat $(4 \mathrm{~m}<x<9.5 \mathrm{~m})$. En revanche, elle subit une légère augmentation et atteint sa valeur maximale au pied de la pente dans le cas du train d'ondes de Pierson-Moskowitz. Ceci pourrait être une manière pour compenser la dissipation énergétique dans E2. En aval de déferlement $(x>x b)$, une dissipation rapide et importante est observée dans le cas de tous les trains d'ondes étudiés. Ce constat montre que le déferlement a un impact immédiat sur l'énergie spectrale dans la région E1. En ce qui concerne l'énergie dans la région de transfert E2, la région où le changement spectral est le plus perceptible, elle diminue d'une manière progressive et considérable (entre $10 \%$ et 30\%). Les dissipations énergétiques dans cette région sont reliées à deux phénomènes : la dissipation visqueuse liée au fond et les transferts énergétiques vers les régions E1 et E3. En aval de déferlement, un transfert énergétique inverse de E3 vers E2 a été observé. Ceci peut être le résultat d'un transfert énergétique réversible. De surcroît, l'énergie 


\section{XVİ̀mes Journées Nationales Génie Côtier - Génie Civil \\ Le Havre 2020}

spectrale dans la région des hautes fréquences E3 augmente légèrement sur le fond plat $(4 \mathrm{~m}<x<9.5 \mathrm{~m})$. En aval du déferlement $(x>x b)$, l'énergie dans cette région commence à diminuer jusqu'à s'annuler. Cette constatation est en bon accord avec les résultats obtenus dans les travaux de TIAN et al., (2011) qui ont démontré expérimentalement que la zone des brisants est marquée par une perte énergétique dans les composantes hautes fréquences $\left(1.5 f_{p}<f<2.5 f_{p}\right)$. Nous avons aussi remarqué une légère augmentation de l'énergie (de $8 \%$ à 12\%) dans la région des basses fréquences (E4). Cet accroissement peut s'expliquer par un éventuel transfert d'énergie de E1 vers E4. Les auteurs qui ont réussi a démontré ceci, ont expliqué cette augmentation par un transfert énergétique depuis les régions des hautes fréquences $\left(f>0.9 f_{p}\right)$ qui s'arrête avec le déferlement de la vague. Ces résultats sont qualitativement similaires de point de vue qualitatif pour les quatre trains d'ondes étudiés. Par conséquent, le choix des limites des régions fréquentielles basé sur la méthode expliquée dans ABROUG et al., (2020) est bien justifié.

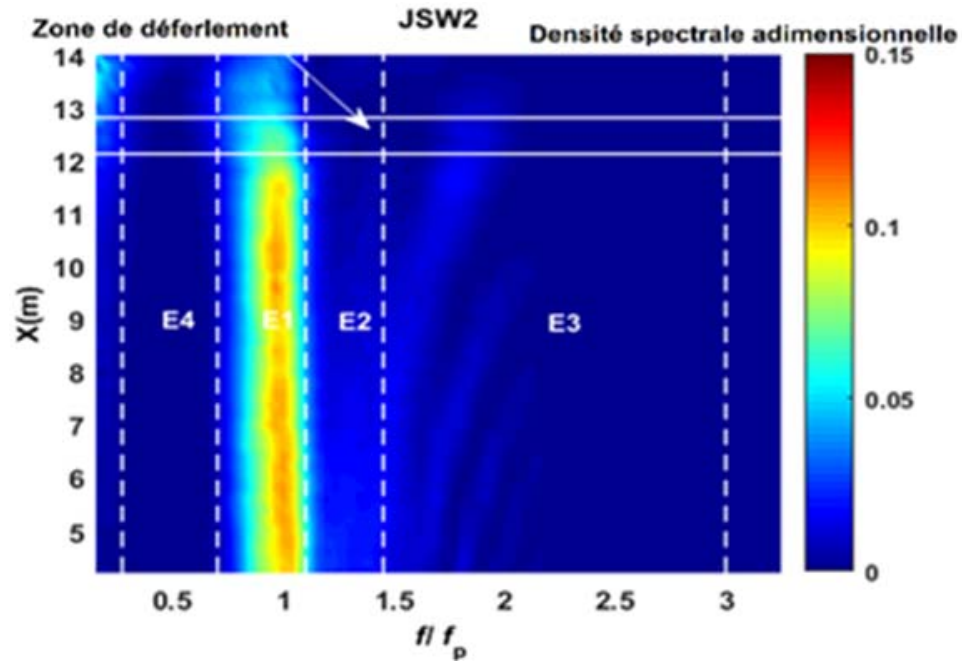

Figure 3. Spectrogramme d'un train d'onde de JONSWAP.

\subsection{Interactions non-linéaires}

Pour un triplet de fréquences donné $\left(f_{1}, f_{2}, f_{1}+f_{2}\right)$, le paramètre de bicohérence varie de 0 pour une interaction non-linéaire non existante à 1 pour une interaction non-linéaire maximale. Cette méthode n'a jamais été utilisée pour traiter les trains d'ondes gaussiens et non-gaussiens se propageant dans une eau de profondeur intermédiaire vers la zone côtière. Dans cet article nous allons nous contenter de présenter le résultat de l'évolution spatiale de la bicohérence dans le cas d'un train d'onde gaussien. 
Thème 1 - Hydrodynamique côtière
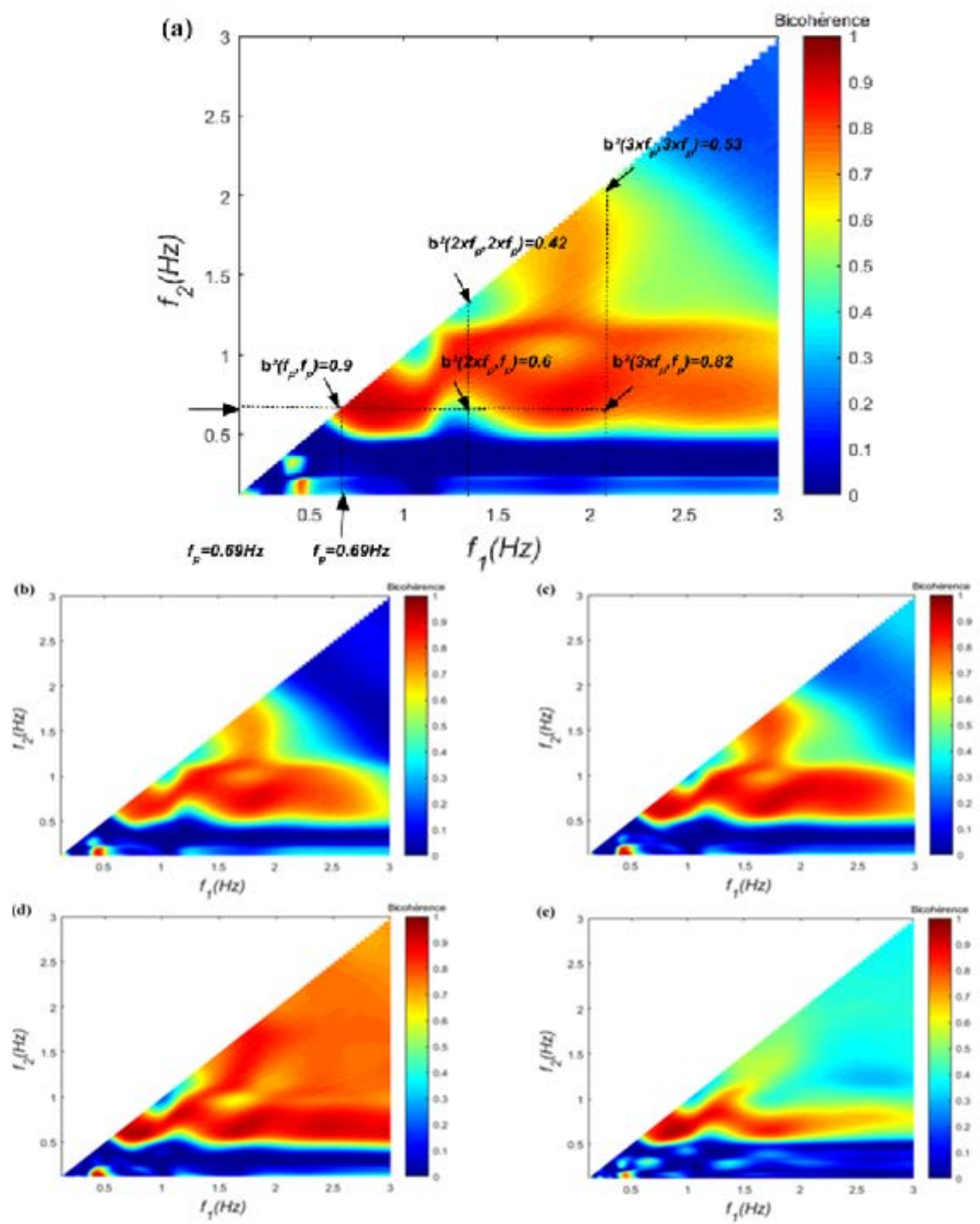

Figure 4. Exemple d'évolution spatiale de la bicohérence entre les harmoniques dans le cas d'un train d'ondes gaussien à 5 différentes sections le long du canal.
(a) $: x=4 m(h=0.3 m)$,
(b) : $x=9.5 \mathrm{~m}(h=0.3 \mathrm{~m})$,
(c) : $x=11 \mathrm{~m}(\mathrm{~h}=0.24 \mathrm{~m})$,
(d) : $x=12 \mathrm{~m}(\mathrm{~h}=0.2 \mathrm{~m})$,
(e) $: x=14 m(h=0.12 m)$. 


\section{XVI'èmes Journées Nationales Génie Côtier - Génie Civil \\ Le Havre 2020}

La figure 4 montre l'évolution spatiale de la bicohérence pour un train d'ondes Gaussien GW1 dans 5 sections différentes le long du canal. Nous précisons ici que la zone de déferlement de ce train d'ondes est située entre $x=11.72 \mathrm{~m}$ et $x=12.70 \mathrm{~m}$. Au cours de la propagation, nous pouvons voir que le nombre de fréquences impliquées dans le processus de focalisation augmente au fur et à mesure.

En $x=4 \mathrm{~m}$, nous pouvons constater que les interactions les plus intenses dans le processus de focalisation sont en majorité aux alentours de la fréquence de pic $f_{p}=0.69 \mathrm{~Hz}$ (voir figure 4a). Par exemple, $b^{2}(0.690 .69)=0.90$ indique la présence d'une interaction forte entre $f_{p}$ et $f_{p}$ couplées avec la fréquence $2 . f_{p}=1.38 \mathrm{~Hz}$. Les résultats expérimentaux ont montré que la bicohérence reste relativement constante le long du fond plat révélant une faible interaction entre les composantes fréquentielles autres que $f_{p}\left(b^{2}\left(f_{1}, f_{2}\right) \sim 0\right)$ (voir figure $4 b)$.

Cependant, une augmentation significative de la bicohérence est observée avec le début du shoaling et qui continue pour atteindre son niveau maximal en $x=x b$. En s'approchant des côtes, la non-linéarité du train d'ondes devient de plus en plus importante et le caractère dispersif devient moins important. Avec le début de déferlement (11.72 $\mathrm{m}<x=12 \mathrm{~m}$; figure $\mathrm{d})$, le nombre de fréquences participant au processus de focalisation atteint son maximum.

En aval du déferlement, ce paramètre décroit drastiquement (voir figure 4e). Ce constat est dû principalement à la décroissance drastique des composantes hautes fréquences qui accompagne le déferlement du train d'ondes (approche spectrale).

Nous pouvons par conséquent conclure que l'augmentation des interactions entre les composantes fréquentielles est parmi les raisons principales du déferlement des trains d'ondes dans les eaux peu profondes.

\section{Références bibliographiques}

ABROUG I., ABCHA N., DUTYKH D., JARNO A., MARIN F. (2020). Experimental and numerical study of the propagation of focused wave groups in the nearshore zone. Physics Letters A, Vol. 384 (6), 126144. https://doi.org/10.1016/j.physleta.2019.126144 BECQ-GIRARD., FORGET. P., BENOIT M. (1999). Nonlinear propagation of unidirectional wave fields over varying topography. Coastal Engineering, Vol. 38 (2), pp 91-113. https://doi.org/10.1016/S0378-3839(99)00043-5

CHUNG J., POWERS EJ. (1998). The statistics of wavelet-based bicoherence. Proceedings of the IEEE-SP International Symposium on Time-Frequency and TimeScale Analysis, (Cat. No.98TH8380), Pittsburgh, PA, USA, pp 141-144. https://doi.org/10.1109/TFSA.1998.721381

DONG G., MA Y., PERLIN M., MA X., YU B., XU J. (2008). Experimental study of wave-wave nonlinear interactions using the wavelet-based bicoherence. Coastal engineering, Vol 55, pp 741-752. https://doi.org/10.1016/j.coastaleng.2008.02.015 


\section{Thème 1 - Hydrodynamique côtière}

ELDEBERKY Y. (1996). Nonlinear transformation of wave spectra in the nearshore zone. PhD. Thesis, published as communication on Hydraulic and Geotechnical Engineering, Delft University of technology, Report No. 96-4, 203 p.

ELGAR S., GUZA R T. (1988). Statistics of bicoherence. IEEE transactions of acoustics, speech and signal processing, Vol. 36, pp 1667-1668. https://doi.org/10.1109/29.7555

FARGE M. (1992). Wavelet transforms and their applications to turbulence. Annual review of fluid mechanics, Vol. 24, pp 395-457. https://doi.org/10.1146/annurev.fl.24.010192.002143 LARSEN Y., HANSSEN A., PECSELI H. L. (2001). Analysis of non-stationary mode coupling by means of wavelet-bicoherence. Proceedings IEEE, International conference on acoustics, speech, and signal processing, Proceedings (Cat. No.01CH37221), Salt Lake City, UT, USA Vol. 6, pp 3581-3584. https://doi.org/10.1109/ICASSP.2001.940616

LIU D., MA Y., DONG G., PERLIN M. (2016). Detuning and wave breaking during nonlinear surface wave focusing. Ocean Engineering, Vol 113, pp 215-223. https://doi.org/10.1016/j.oceaneng.2015.12.048

MADSEN P. A., SORENSEN O. R. (1993). Bound waves and triad interactions in shallow water. Ocean engineering, Vol. 20, pp 359-388. https://doi.org/10.1016/00298018(93)90002-Y

MA Y., DONG G., LIU S., ZANG J. (2010). Laboratory study of unidirectional focusing waves in intermediate depth water. Journal of engineering mechanics, Vol. 136, pp 7890. https://doi.org/10.1061/(ASCE)EM.1943-7889.0000076

TIAN Z., PERLIN M., CHOI W. (2011). Frequency spectra evolution of two-dimensional focusing wave groups in finite water depth water. Journal of fluid mechanics, Vol. 688, pp 169-194. https://doi.org/10.1017/jfm.2011.371

TORRENCE C., COMPO G P. (1998). A practical guide to wavelet analysis. Bulletin of American meteorology society, Vol. 79, pp 61-78. https://doi.org/10.1175/15200477(1998)079<0061:APGTWA>2.0.CO;2

TOUBOUL J., KHARIF C. (2006). On the interaction of wind and freak waves due to modulational instability. Physics of fluids, Vol. 18 (10), pp 108103.1-108103.4. https://doi.org/10.1063/1.2374845

TOUBOUL J., KHARIF C. (2015). Les vagues scélérates : mécanismes de génération et prédictibilité. La météorologie, Vol. 90, pp 62-68. https://doi.org/10.4267/2042/56840

VAN MILLIGEN B P., SANCHEZ E., ESTRADA T., HILDALGO C., BRANAS B., CARRERAS B., GARCIA L. (1995). Wavelet bicoherence : a new turbulence analysis tool. Physics of Plasmas, Vol. 2 (8), pp 3017-3032. https://doi.org/10.1063/1.871199

WHITTAKER C. N., FITZGERALD C. J., RABY A. C., TAYLOR P. H., ORSZAGHOVA J., BORTHWICK A. G. L. (2017). Optimisation of focused wave group runup on plane beach. Coastal engineering, Vol. 121, pp 44-55. https://doi.org/10.1016/j.coastaleng.2016.12.001 